\title{
Quantum superchemistry in an output coupler of coherent matter waves
}

\author{
H. Jing ${ }^{1,2}$ and J. Cheng ${ }^{3}$ \\ ${ }^{1}$ Department of Physics, The University of Arizona, 1118 East 4th Street, Tucson, AZ 85721 \\ ${ }^{2}$ State Key Lab of Magnetic Resonance and Atomic and Molecular Physics, \\ Wuhan Institute of Physics and Mathematics, CAS, Wuhan 430071, P.R. China \\ ${ }^{3}$ Lab for Quantum Optics, Shanghai Institute of Optics and Fine Mechanics, \\ Chinese Academy of Sciences, Shanghai 201800, P.R.China
}

\begin{abstract}
We investigate the quantum superchemistry or Bose-enhanced atom-molecule conversions in a coherent output coupler of matter waves, as a simple generalization of the two-color photo-association. The stimulated effects of molecular output step and atomic revivals are exhibited by steering the rf output couplings. The quantum noise-induced molecular damping occurs near a total conversion in a levitation trap. This suggests a feasible two-trap scheme to make a stable coherent molecular beam.
\end{abstract}

PACS numbers: 42.50.-p, 03.70.+k, 03.75.Pp

The realization of Bose-Einstein condensates (BEC) in ultracold atomic gases has led to a profound revolution in modern physics, from low-temperature physics to atom optics [1]. Recently there has been many interests in creating a molecular BEC (MBEC) via a magnetic Feshbach resonance [2-3] or an optical photo-association (PA) technique [4] within an atomic BEC. As an atom-optics analog of the simplest nonlinear quantum optical system (second-harmonic generation) [5], the quantum properties of the hybrid BEC-MBEC have been extensively studied. For example, Herbig et al. [2] created a pure molecular quantum gas spatially separated from its atomic partner by adding a magnetic levitation field to an ordinary Feshbach resonance technique within a cesium BEC. Winkler et al. [4] experimentally studied the coherent two-color PA process, a process first dubbed by Heinzen et al. as superchemistry or Bose-enhanced free-bound transition [6]. Also, Hope and Olsen [7] predicted the onset of large molecular damping due to the quantum noises or spontaneous processes in the quantum superchemistry where the mean-field (MF) approach breaks down for certain parameters.

The coherent PA features a weak-light-induced resonant intra-mode coupling and subsequent diatomic MBEC at low temperatures as the microkelvin range, and the practical efficiency of molecules generation is limited by radiative decay and particle collisions [7]. The versatile technique of Feshbach resonance, in which the spin of one of two colliding atoms flips in a magnetic field, is widely exploited in an optical trap [2] to create a MBEC with high efficiency [3], along with other important applications like the generations of matter-wave bright solitons [8], which are made possible by tuning the atomic interactions. By combining these magneto-optic techniques, many theoretical schemes have been proposed to achieve an efficient and stable conversion of atoms to molecules in a trapped BEC [9], by minimizing the impact of negative factors like the MF shift and the vibrational relaxations.

Recently, Zhang et al. [10] proposed a Feshbach resonance technique applied to a travelling atomic beam instead of an optically confined BEC to study atomic filamentation and pair correlations. Then similarly, it is natural to extend to study the quantum superchemistry in an atom-laser output coupler. In this article, we consider the concrete example of the MIT rf output coupler which first realized a pulsed atom laser to address the question of whether the presence of an optical sheet tuned near the PA resonance immediately below the magnetic trap [11] can lead to new and different quantum dynamical effects in the output matter waves. The quantum superchemistry of the out-coupled hybrid matter 
waves is studied by using the powerful tool of positive- $P$ representation in quantum optics $[7,12]$. We identify the stimulated effects of the molecular output step and atomic revivals by steering the rf output couplings. The coherent conversion of atoms to molecules is robust, with the noise-induced molecular damping occurring near the total conversion. If these effects are typical, then the quantum superchemistry in an atom laser can be tunable and potentially useful for, e.g., making a continuous molecule laser. A practical realization of this scheme could be conceived by exploiting the levitationtrap technique of Herbig et al. [2] to collect and then separate the molecules from the output hybrid atomic-molecular beam.

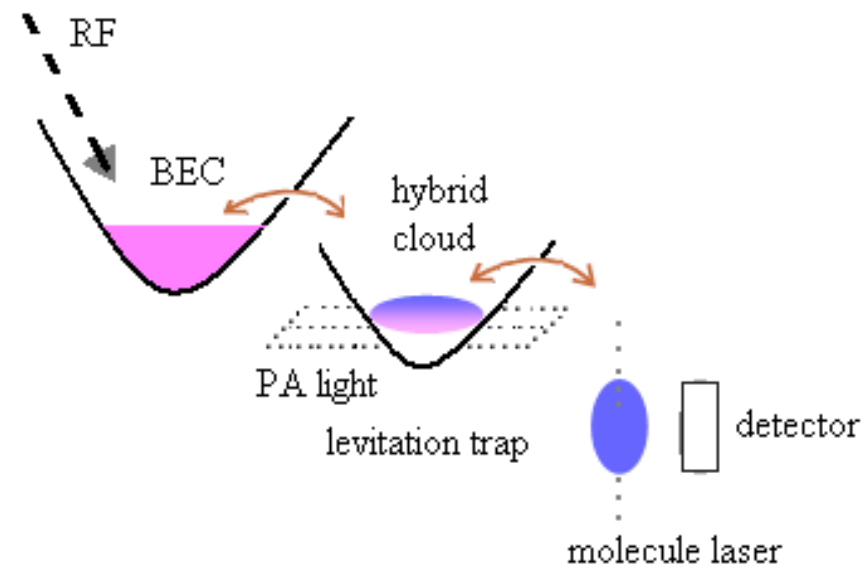

FIG. 1: (Color online) Schematic diagram of (non-Arrhenius) superchemistry process in a matter wave coupler. The external RF field provides an effective atomic tunnelling between the two magnetic traps. This is followed by molecule generation in the second (levitation) trap where the PA happens in an initial vacuum. The hybrid BEC-MBEC collected in the levitation trap can also be separated to form a coherent molecular beam [2, 11].

As illustrated in Fig. 1, we consider for simplicity that a large number of Bose-condensed atoms with two states is condensed initially in the trapped state $|1\rangle$. State $|2\rangle$, which is typically unconfined, is coupled to state $|1\rangle$ by the rf field tuned near the $|1\rangle \rightarrow|2\rangle$ transition. The coherent PA process occurs in a levitation trap which is initially in a vacuum. The resonantly enhanced atom-molecule coupling then coherently converts [4] the output atoms into molecules in the single-state $|3\rangle$. The Bose enhancement of the transition is essential since the actual PA coupling is weak due to the low FranckCondon factors. Generally, to study the PA process one first uses a single-mode approach (SMA) [13] to understand some main features, especially for a one-dimensional BEC with small kinetic energy or a coupling pulse of short duration. This approximation is further evaluated by the recent work of Winkler et al., who found that their two-color PA experiment can be well described by a SMA [4]. The spatial structure analysis can be an important correction especially for large variations in density [7]. Here we assume that both the atomic and molecular outputs form controllable monochromatic beams and focus on the couplings of different components. The boson annihilation operators for trapped and output atoms, and subsequent molecules are denoted, respectively, by $\hat{a}_{1}, \hat{a}_{2}$ and $\hat{b}$. Defining the rf (Rabi) and PA strengths as $G^{\prime}$ and $\gamma$, the effective Hamiltonian in a rotating frame is then $(\hbar=1)$

$$
\hat{H}=-\delta \hat{b}^{\dagger} \hat{b}+G^{\prime}\left(\hat{a}_{1}^{\dagger} \hat{a}_{2}+a_{2}^{\dagger} \hat{a}_{1}\right)+\gamma\left(\hat{a}_{2}^{\dagger 2} \hat{b}+\hat{b}^{\dagger} \hat{a}_{2}^{2}\right)+\lambda_{a}\left(\hat{a}_{1}^{\dagger 2} \hat{a}_{1}^{2}+\hat{a}_{2}^{\dagger 2} \hat{a}_{2}^{2}\right)+\lambda_{b} \hat{b}^{\dagger 2} \hat{b}^{2}
$$

where the molecular decaying into unimportant states can be included by a non-Hermitian term in the 
optical detuning, e.g., $\delta \rightarrow \delta-\frac{1}{2} i \Gamma$ [13], with $\Gamma$ bing proportional to the total molecular decay rate. The attractive intra-mode collisions are ignored in order to compare with the trapped BEC case [7]. Our model is essentially different from the well-known two-color PA $[4,6-7,13]$ which, as will be shown later, also can be included by our model under the adiabatic approximation. Note that two different regimes may exist in our system, the regime of atomic tunnelling, followed by molecule generation in the second trap, and a propagating regime of separated matter waves (at the time corresponding to the stable molecular step as described later). Our study focuses on the first regime only and thus we ignored the kinetic-energy terms in the Hamiltonian. For the long-distance propagating regime, Zhang et al. already predicted some novel effects for a travelling hybrid beam [8, 10, 18].

The full quantum effects are included by using the positive- $P$ representation of quantum optics [12]. The initial trapped state $|1\rangle$ is taken as a coherent state and the other two relevant states are initially in a vacuum. It is easy to represent them as $\delta$ functions but much difficult for an initial number state. Using the standard correspondence relations between $\left\{\hat{a}_{1}, \hat{a}_{1}^{\dagger}, \hat{a}_{2}, \hat{a}_{2}^{\dagger}, \hat{b}, \hat{b}^{\dagger}\right\}$ and $\left\{\alpha_{1}, \alpha_{1}^{\dagger}, \alpha_{2}, \alpha_{2}^{\dagger}, \beta, \beta^{\dagger}\right\}$, we find the following set of coupled $c$-number Itô stochastic differential equations $(\delta=0)$ :

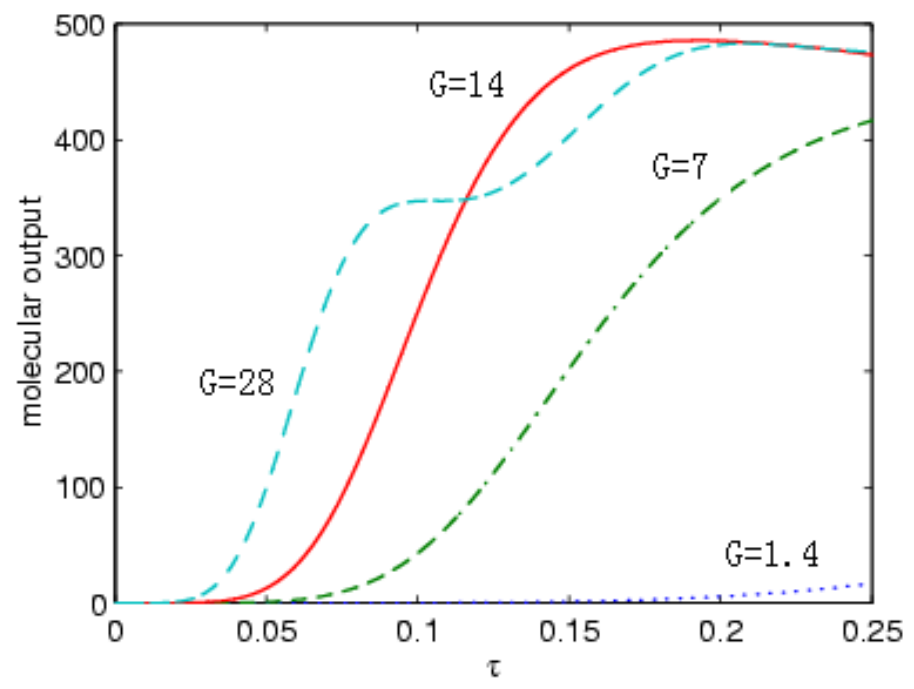

FIG. 2: (Color online) The molecular output numbers for different rf couplings (scaled by the PA strength, i.e., $\left.G=G^{\prime} / \gamma\right)$. The scaled time $\tau(=\gamma t)$ is dimensionless and the value 0.145 here corresponds to half of the maximum evolution time $1 \mu \mathrm{s}$ used by Hope et al. [7]. The parameters used here are described in the text.

$$
\begin{aligned}
\frac{d \alpha_{1}}{d t} & =-i G^{\prime} \alpha_{2}-2 i \lambda_{a} \alpha_{1}^{+} \alpha_{1}^{2}+\sqrt{-2 i \lambda_{a} \alpha_{1}^{2}} \eta_{1}(t), \\
\frac{d \alpha_{1}^{+}}{d t} & =i G^{\prime} \alpha_{2}^{+}+2 i \alpha_{1} \lambda_{a} \alpha_{1}^{+2}+\sqrt{2 i \lambda_{a} \alpha_{1}^{+2}} \eta_{2}(t), \\
\frac{d \alpha_{2}}{d t} & =-i G^{\prime} \alpha_{1}-2 i \gamma \alpha_{2}^{+} \beta-2 i \lambda_{a} \alpha_{2}^{2} \alpha_{2}^{+}+\sqrt{-2 i \gamma \beta-2 i \lambda_{a} \alpha_{2}^{2}} \eta_{3}(t), \\
\frac{d \alpha_{2}^{+}}{d t} & =i G^{\prime} \alpha_{1}^{+}+2 i \gamma \alpha_{2} \beta^{+}+2 i \lambda_{a} \alpha_{2}^{+2} \alpha_{2}+\sqrt{2 i \gamma \beta^{+}+2 i \lambda_{a} \alpha_{2}^{+2}} \eta_{4}(t), \\
\frac{d \beta}{d t} & =-i \gamma \alpha_{2}^{2}-2 i \lambda_{b} \beta^{+} \beta^{2}+\sqrt{-2 i \lambda_{b} \beta^{2}} \eta_{5}(t), \\
\frac{d \beta^{+}}{d t} & =i \gamma \alpha_{2}^{+2}+2 i \lambda_{b} \beta \beta^{+2}+\sqrt{2 i \lambda_{b} \beta^{+2}} \eta_{6}(t),
\end{aligned}
$$


where the $\eta_{i}$ are real Gaussian noise terms with the correlations $\overline{\eta_{i}(t)}=0$ and $\overline{\eta_{i}(t) \eta_{j}\left(t^{\prime}\right)}=\delta_{i j} \delta\left(t-t^{\prime}\right)$. The $c$-number variables are not complex conjugate except in the mean of a large number of stochastic trajectories. Eq.(2) can reduce to the MF case in the noiseless limit. By solving it, any normally ordered quantity can be calculated, including the quantum statistics of the resultant fields which are difficult to be probed in the experiment [4]. Here the quantities of interest are the occupations in each of three states, especially the molecular output number. The numerical stochastic integration is performed by averaging selected moments of the fields over a sufficiently large sample of trajectories. The initial atoms number is assumed as 1000 and, to compare with the trapped case [7], we set $\lambda_{a}=\lambda_{b}$ and use the mass and scattering length of ${ }^{87} \mathrm{Rb}$ atoms.

We note that the scattering length of Rubidium molecules and even the attractive (instead of repulsive) atom-molecule interactions are now known experimentally. In fact, it is straightforward to use some different collision values in our numerical computation. (We have actually done this, and found that the difference between the positive- $P$ and mean-field (MF) results can be even greater in that case.) However, in order to compare with the results of Hope and Olsen [7], our present study adopts their parameters, including the PA light strength and the assumption of equal particle collisions. As shown in Fig.2, the molecular output numbers generally increase with respect to the scaled rf couplings; but for stronger rf coupling $(G=28)$, we observe a molecular output step, i.e., the molecular number increases rapidly at first but then much slowly. This has a simple explanation. The rf coupling can induce a perfect atomic oscillation without the PA [14] which, however, is deformed as an atomic revival here. For a wide regime of rf couplings there is a period of stable molecular output before reaching a total conversion, with a similar time scale as in the trapped BEC case [7].

The new feature of the present scheme, as confronted in earlier analysis, is that the coherent PA process starts not directly in a trapped BEC, but in an output atomic field that is initially in a vacuum. We note that the formally similar SMA model studied by Calsamiglia et al. [13] on two-color PA within a Fock-state atomic BEC contains a linear molecular coupling and their threemode Hamiltonian was reduced by eliminating the intermediate molecular mode under large evolution frequency approximation. We focus here, however, on the role of atomic tunnelling on the molecular generations in which the intermediate atomic mode cannot be adiabatically eliminated [13].

To compare the fully quantum and MF results, we plot the Fig. 3 to show the atomic and molecular output numbers for different rf couplings. The MF results are simply from the noiseless limit of Eq.(2). We see that, for weaker rf coupling, the molecular generation is very similar as in the trapped BEC. But for stronger rf coupling, an effect of molecular output step is observed around the minimum atomic number, with an atomic revival instead of the perfect oscillation in two-state stimulated transition [14]. This provides clear evidence of a long-range coherence effect. In contrast, the PA process in a thermal atomic beam would not produce similar step or revival, because the phases associated with the individual atom-molecule conversion are random in that case. This is also different from the long-time behaviors in a trapped BEC. These results, qualitatively valid beyond the zero-dimensional model, clearly show the roles of the linear rf output coupling and the nonlinear PA interaction.

The fully quantum approach is effectively the same as the MF approach before reaching full atommolecule conversion [7]. But their differences can be largely amplified in a complex three-dimensional analysis by taking into account of many non-ideal factors with the long-time evolutions [7,15]. Thus 

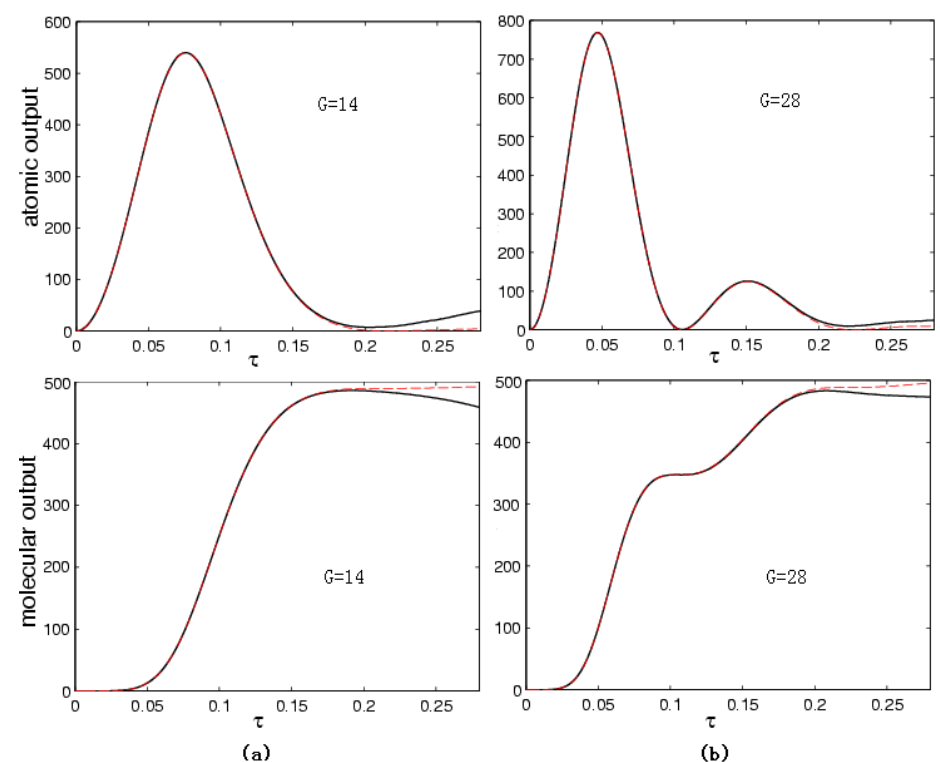

FIG. 3: (Color online) The atomic and molecular output numbers for different rf couplings. The black lines show the fully quantum results while the red dotted lines are that of MF approach. For lower rf coupling (a): the atomic revival is shown only by considering the quantum noise [7]; but for stronger rf coupling (b): more atomic revivals can appear in the output hybrid beams. The first revival is exhibited even by MF approach. Around the minimum atomic number, there is also an interesting output step effect for the molecules.

it might be of some interests for an atom-laser-based experiment, instead of a tightly confined BEC, to observe the molecular damping first predicted by Hope and Olsen [7] in quantum superchemistry.

Our model also can include the quasi-bond-bond molecular coupling $\Omega\left(\hat{g}^{\dagger} \hat{b}+\hat{b}^{\dagger} \hat{g}\right)$ by applying a pair of short, overlapping laser pulses, with $\hat{g}$ denoting the stable molecular mode, as a generalization of the familiar Raman two-color PA process [7,13]. In fact, if we ignore the collisions in the rarely populated intermediate molecular mode and take $|\delta|$ as the largest frequency in the system, we can set $\dot{\hat{b}} / \delta=0$ and obtain $\hat{b} \simeq\left(\Omega \hat{g}+\gamma \hat{a}_{2}^{2}\right) / \delta$. This amounts to adiabatic elimination of the excited-molecular mode and yields an effective interacting Hamiltonian which still has the form of Eq. (1) with the following substitution (neglecting the free motions and the tunable atomic collisions in the trap)

$$
\hat{b} \rightarrow \hat{g}, \quad G^{\prime} \rightarrow G^{\prime}, \quad \gamma \rightarrow \chi=\gamma \Omega / \delta, \quad \lambda_{a} \rightarrow \lambda_{a}+\gamma^{2} / \delta, \quad \lambda_{b} \rightarrow \lambda_{g} .
$$

Hence, for $\gamma=145 \mathrm{KHz}, \Omega=10 \mathrm{GHz}$ and $\delta=1000 \Omega$, we have a much smaller effective PA strength $\chi=145 \mathrm{~Hz}$ [7]. Due to $G=\eta G^{\prime} / \gamma$ and $\eta=\delta / \Omega$, a large scaled $\mathrm{rf}$ coupling requires a higher detuning. The maximum strength here is chosen as: $G^{\prime} \simeq 4 \mathrm{KHz}<4 \sqrt{6} \mathrm{KHz}$, in order to avoid the output shutdown effect for an atom laser due to the density fluctuations in the beam and the losses to other states [16]. This, together with the molecular damping, also sets an upper limit to the flux of a molecule laser. The possible noise suppression due to, e.g., the dimer formation will be probed elsewhere.

In summary, we first reported the results of quantum superchemistry or Bose-enhanced quantum dynamics in an output coupler of coherent matter waves. We have showed that a step in the growth of the molecular output and the atomic revivals can appear by steering the external rf fields, and that the coherent atom-molecule conversion is robust before the noise-induced molecular damping occurring 
near a total conversion. It will add an experimental level of difficulty to observe both two types of atomic revivals due to the stimulated or the spontaneous processes, but with the current progress in coherent coupling and PA of condensed atoms [1,4,11], some possibilities are certainly open. If the effects of molecular output step and rf-induced atomic revivals are typical, then the superchemistry process in an atom laser is tunable and very useful for achieving a stable output of molecules. The technique of Herbig et al. [2] and even a collision-shift-compensated PA light with frequency chirp [9] also could be applied to the hybrid beams in making, e.g., a continuous molecule laser [11].

Our studies can be readily generalized to, e.g., a Raman output coupler [17]. And the SMA is suitable for large couplings of different components. In fact, the recent results of coherent PA experiment of Winkler et al. are well described by the SMA [4]. We note that, for the long-distance propagating regime, by using the MF nonlinear Schrödinger equation, some novel effects were also predicted by Zhang et al. for a travelling hybrid beam with large attractive atom-molecule interactions, such as the nonlocal behaviors or solitons of the output fields $[8,10,18]$. A three-dimensional correction can be made similarly by using some extremely complicated method and fully including many nonideal factors, such as the phase diffusion of the BEC, the inelastic inter-mode scattering and even the threebody losses at higher densities (see, e.g., Refs. [7,15,19-20]). Finally, the role of quantum statistics of the initial atoms in our system will also be interesting and deserves further study.

H. J. thanks P. Meystre for his very useful discussions. This work was supported by Natural Science Foundation of China (10304020, 10404031), Wuhan Sunshine Plan and Shanghai Rising-Star Program.

[1] J. R. Anglin and W. Ketterle, Nature (London) 416, 211 (2002); P. Meystre, Atom Optics (SpringerVerlag, 2001); F. Dalfovo, S. Giorgin, L. P. Pitaevskii and S. Stringari, Rev. Mod. Phys. 71, 463 (1999).

[2] J. Herbig, et al., Science 301, 1510 (2003); E. A. Donley, et al., Nature (London) 417, 529(2002); S. Inouye et al., Nature (London) 392, 151 (1998); J. L. Roberts, et al., Phys. Rev. Lett. 86, 4211 (2001).

[3] E. Timmermans, et al., Phys. Rep. 315, 199 (1999); F. A. van Abeelen and B. J. Verhaar, Phys. Rev. Lett. 83, 1550 (1999); S. J. J. M. F. Kokkelmans, H. M. J. Vissers and B. J. Verhaar, Phys. Rev. A 63, 031601 (2001); M. Holland, J. Park and R. Walser, Phys. Rev. Lett. 86, 1915 (2001).

[4] K. Winkler, et al., Phys. Rev. Lett. 95, 063202 (2005); R. Wynar, et al., Science 287, 1016 (2000); M. Mackie, et al., Phys. Rev. Lett. 843803 (2000); N. Vanhaecke, et al., Phys. Rev. Lett. 89, 063001 (2002).

[5] M. O. Scully and M. S. Zubairy, Quantum Optics (Cambridge University, 1997).

[6] D.J. Heinzen, et al., Phys. Rev. Lett. 84, 5029 (2000).

[7] J. J. Hope and M. K. Olsen, Phys. Rev. Lett. 86, 3220 (2001); J. J. Hope, Phys. Rev. A 64, 053608 (2001); J. J. Hope, M. K. Olsen, and L. I. Plimak, Phys. Rev. A 63, 043603 (2001).

[8] E. Donley et al., Nature (London) 412, 295 (2001). M. Mackie, K.-A. Suominen and J. Javanainen, Phys. Rev. Lett. 89, 180403 (2002); L. Khaykovich, et al., Science 296, 1290 (2002); P. G. Kevrekidis, et al., Phys. Rev. Lett. 90, 230401 (2003).

[9] H.-Y. Ling, H. Pu and B. Seaman, Phys. Rev. Lett. 93, 250403 (2004); J. Cheng, et al., Phys. Rev. A 73, 035601 (2006); F. H. Mies, E. Tiesinga and P. S. Julienne, Phys. Rev. A 61, 022721 (2000).

[10] W.-P. Zhang, et al., Phys. Rev. Lett. 90, 140401 (2003).

[11] M.-O Mewes, et al., Phys. Rev. Lett 78, 582 (1997); A. P. Chikkatur, et al., Science 296, 2193 (2002).

[12] P. D. Drummond and C. W. Gardiner, J. Phys. A 13, 2353 (1980).

[13] J. Calsamiglia, M. Mackie and K.-A. Suominen, Phys. Rev. Lett. 87, 160403 (2001); A. Vardi, et al., Phys. Rev. A 64, 063611 (2001); Y. Wu and R. Coté, Phys. Rev. A 65, 053603 (2002).

[14] H. Jing, J.-L. Chen and M.-L. Ge, Phys. Rev. A 63, 015601 (2001).

[15] J. Stenger, et al., Phys. Rev. Lett. 82, 2422(1999); N. R. Claussen, et al., Phys. Rev. Lett. 89, 010401 (2002); T. Mukaiyama, et al., Phys. Rev. Lett. 92, 180402 (2004).

[16] N. P. Robins, et al., Phys. Rev. A 72, 031606(R) (2005); N. P. Robins, et al., 69, 051602(R) (2004); N. P. Robins, et al., Phys. Rev. Lett. 96, 140403 (2006). 
[17] S. A. Haine, M. K. Olsen and J. J. Hope, Phys. Rev. Lett. 96, 133601 (2006).

[18] K.V. Kheruntsyan, M. K. Olsen, and P. D. Drummond, 95, 150405 (2005); T. G. Vaughan, K. V. Kheruntsyan, and P. D. Drummond, Phys. Rev. A 70, 063611 (2004).

[19] M. Mackie, Phys. Rev. Lett. 91, 173004 (2003).

[20] V. A. Yurovsky and A. Ben-Reuven, Phys. Rev. A 70, 013613 (2004). 